\title{
The Dose-Response Effect of Medical Exercise Therapy on Impairment in Patients with Unilateral Longstanding Subacromial Pain
}

\author{
Håvard Østerås ${ }^{*}, 1$ and Tom Arild Torstensen ${ }^{2}$ \\ ${ }^{1}$ Sør-Trøndelag University College, Faculty of Health Education and Social Work, Department of Physical Therapy, \\ N-7004 Trondheim, Norway \\ ${ }^{2}$ Holten Institute, Lidingö, Sweden
}

\begin{abstract}
Objectives: The primary aim of this study was to investigate the effect of medical exercise therapy in shoulder impingement patients, along with possible correlations between impairment variables.

Study Design: A prospective unblended randomized clinical trial.

Methods: Over four months, 61 participants were randomly assigned into a high-graded exercise therapy group (HD) $(n=31)$ and into a low-graded exercise therapy group (LD) $(n=30)$. Prognostic variables were similar between the groups at baseline. Five (8\%) patients dropped out during the treatment period, and another four $(6 \%)$ dropped out before followup. Pain was a composite score of a visual analogue scale (VAS). Isometric strength was measured during four resisted break tests on the shoulder. Function was measured by means of a functional assessment questionnaire (Shoulder Rating Questionnaire, SRQ). Both groups trained three times per week for twelve weeks, with tests pre- and posttraining and six months follow-up.

Results: The HD group achieved significantly $(\mathrm{p}<0.05)$ better outcome effects than the LD group for pain, range of motion, isometric functional strength and function, but both groups increased function from pretest to posttest.

Conclusions: In patients with uncomplicated subacromial pain syndrome, medical exercise therapy is an efficient treatment alternative, where high-grade doses should be emphasized. A major limitation is that the measurements were not undertaken by another person than the treating physiotherapists.
\end{abstract}

Keywords: Shoulder, rehabilitation, impingement, physical therapy, dose-response, muscle function.

\section{INTRODUCTION}

Tendinitis of the rotator cuff and shoulder impingement syndrome are considered to be the most common intrinsic causes of shoulder pain and disability [1,2]. The prevalence of shoulder pain among adults under 70 years of age is 7 $27 \%$, whereas this figure is between $13.2-26 \%$ among those over 70 [3]. A number of systematic reviews on studies involving shoulder interventions have found little evidence of exercise used in the treatment of shoulder impingement syndrome [e.g. 4]. Furthermore, when exercises have been used, the studies have been criticized for a lack of detailed description of these exercises $[5,6]$.

Inconsistent findings in the literature as to treatment effects of exercise therapy in subacromial pain syndrome might be explained by the overall low intervention doses used. The hypothesis is that there are benefits to be gained by increasing doses of exercise training for patients with subacromial pain. The term "medical exercise training" is as yet little used in physiotherapy. It consists of a progressively graded exercise system developed in Norway in the early

*Address correspondence to this author at the Sør-Trøndelag University College, Faculty of Health Education and Social Work, Department of Physical Therapy, Ranheimsv 10, N-7004 Trondheim, Norway; Tel: +4773 5593 05; Fax: +47 735593 51; E-mail: havard.osteras@hist.no 1960s [7]. The aim is to normalize function by using specific exercises for mobilizing hypomobile areas, and stabilizing exercises for the different areas of the body. By using seven to nine exercises, most patients perform nearly 1000 repetitions during each treatment, possibly influencing such mechanisms as endurance, coordination, and circulation. The grading of the exercises makes it possible to exercise with no (or virtually no) pain.

The goal of the present study was to assess pain, function, muscle strength, and range of motion in patients with shoulder impingement syndrome who have participated in medical exercise training.

\section{MATERIALS AND METHODOLOGY}

\section{Subjects}

Patients between 18 and 60 years of age with unilateral primary shoulder impingement syndrome were recruited from their primary medical doctors. They were consecutively included in the study if they fulfilled the following inclusion criteria; positive subacromial impingement sign (standardized procedure so that the interpretation was consistent from physician to physician; humerus abducted 90 degrees in the scapulae plan, maximal passive inward rotation should give subacromial pain) [8], a minimum of three months since the onset of shoulder symptoms, no previous shoulder surgery, 
normal neck (no neurological signs), elbow, and thoracic spine function with no referred pain from this area to the shoulder, no neurological diseases, no history of shoulder dislocation, subluxation, or fracture, no vestibular or visual disturbances, no adhesive capsulitis, and no chiropractic or physiotherapy treatment within the last six months prior to entering the study. Individuals were also excluded if they had any cardiovascular, respiratory, systemic, or metabolic conditions limiting their ability to participate in the study. Patients showing signs of rotator cuff tears (drop arm test) or other disorders in the glenohumeral joint during the physical examination were then excluded. The included patients were randomly assigned to either a high-dose medical exercise therapy group (HD) or a low-dose exercise therapy group (LD). The randomization procedure was concealed from the experimenters and treating physiotherapist. Another physiotherapist drew a closed envelope with a number of papers corresponding to the number of participants in the two groups. Ethical approval was acquired from the Human Review Committee (Trondheim, Norway) and all participants gave their written consent to participate in the study after receiving written information about it. Three physiotherapists at three different locations were involved in the study, and the same physiotherapist always dealt with the same patients.

All the included patients underwent a one-day testing procedure before and after the three-month experimental period, in addition to a six months follow-up. The tester was not blinded to which intervention the patient received. The patients were informed about the length of the study, about test and treatment procedures, and were told that they could withdraw from the study at any time. Baseline data for all the included patients is presented in Table $\mathbf{1}$.

\section{Measurements}

The primary outcome was a subjective pain score, which was measured with a visual analog scale (VAS) at rest. The VAS has been shown to be a reliable tool for measuring pain [9]. The pain responses were recorded on a $0-100 \mathrm{~mm}$ line used for each test. The extreme limits were marked by perpendicular lines using the verbal descriptors of "no pain" or "worst pain I can imagine". The patients were not shown their previous markings when follow-up measurements were taken.

The secondary outcome was function, measured using a functional assessment questionnaire. The functional outcome measures included the self-completed Shoulder Rating Questionnaire (SRQ) for which uniform instructions were given. The five main components are pain (VAS), pain in specific components, activity in daily living, activity level in sports and leisure, and work ability. Psychometric properties (reliability, validity, and responsiveness to change) of the SRQ have been previously reported for patients with shoulder pathology $[10,11]$. The SRQ was scored using the method described by [10], resulting in scores rating from 17 90 , with higher scores indicating better shoulder function with less shoulder symptoms.

Maximal isometric muscle strength of abduction, flexion, and external and internal rotation was measured using a digital dynamometer [12]. To measure the patients' maximal isometric strength, they were asked to press their arm against the digital dynamometer which was held by a physiotherapist. The MicroFET2, a hand-held dynamometer with a high inter-rater reliability $[13,14]$, measures the force between 0.9-660 N. All tests were performed in a standing position. 1) Shoulder flexion, with the dynamometer fixed distally at the humerus to avoid elbow flexion bias. 2) Shoulder internal rotation, with the elbow flexed 90 degrees and the dynamometer fixed against the distal forearm. 3) Shoulder external rotation, with the elbow flexed 90 degrees and the dynamometer fixed against the distal forearm. 4) Abduction of the arm with the arm 30 degrees abducted, and with the dynamometer resisting further abduction fixed at the distal forearm. Every maximal isometric test was performed as a "break-test", as the physical therapist gave sufficient resistance during the approximately three-second tests so that the patient could not resist the external force.

\section{Treatment Procedures}

The principles of progressive resistance exercise therapy using medical exercise therapy were applied to both exercise groups. Patient history, symptoms, and clinical findings were

Table 1. Mean (SD) Baseline Demographic and Clinical Characteristics of Each Group at Inclusion

\begin{tabular}{|c|c|c|c|c|}
\hline Variables & Mean & SD & Mean & SD \\
\hline Age (years) & 46.1 & 11.2 & 41.8 & 14.5 \\
\hline Height $(\mathrm{cm})$ & 175.8 & 7.4 & 174.1 & 6.3 \\
\hline Mass (kg) & 79.8 & 12.3 & 79.0 & 17.5 \\
\hline \multicolumn{5}{|l|}{ Sex } \\
\hline Men (\%) & 33.1 & & 26.0 & \\
\hline Women (\%) & 22.1 & & 18.8 & \\
\hline Number of treatments & 29.4 & 8.6 & 30.9 & 7.6 \\
\hline
\end{tabular}


the basis for choosing starting positions, range of motion, and weight resistance for the patient being able to do three sets of 30 repetitions. Each exercise was tested using a specific clinical test procedure developed in medical exercise therapy $[15,16]$. Thus, each patient had an individual tailored exercise program. The patient exercised within the comfortable range of motion with normal humeroscapular rhythm, and in the early phase the weight from the pulley apparatus was used to unload some of the weight of the arm, making it possible to perform the high number of repetitions in sets (three sets of 30 repetitions) with good kinetic control. As the patient improved, experiencing less pain, the range of motion and the weight resistance were increased, and the starting position was changed according to the progression ladder developed in medical exercise therapy [16]. The number of repetitions and sets was kept constant for both intervention groups during the treatment period.

Table 2. Intervention in the Two Groups, Showing Difference in Dosage

\begin{tabular}{|l|l|}
\hline \multicolumn{1}{|c|}{ High-Dosage } & \multicolumn{1}{c|}{ Low-Dosage } \\
\hline \hline $\begin{array}{l}\text { 15-20 minutes aerobic } \\
\text { (stationary bike/treadmill) }\end{array}$ & $5-10$ min global aerobic \\
\hline $\begin{array}{l}4 \text { local exercises } \\
3 \text { sets of 30 repetitions }\end{array}$ & 5 local exercises 2 sets of 10 repetitions \\
\hline $\begin{array}{l}10 \text { minutes aerobic } \\
\text { (stationary bike/treadmill) }\end{array}$ & \\
\hline $\begin{array}{l}4 \text { local exercises } \\
3 \text { sets of 30 repetitions }\end{array}$ & \\
\hline $\begin{array}{l}10 \text { minutes aerobic } \\
\text { (stationary bike/treadmill) }\end{array}$ & \\
\hline
\end{tabular}

The patients' history and clinical tests, including muscle tests, specific joint tests, and functional tests, were used to form the basis for choosing the right grading of the exercises, with emphasis on the appropriate weight resistance and range of motion. Patients were instructed that the exercises might result in muscle fatigue but should not increase the shoulder pain significantly. Making the patient exercise in the pain free range of motion also made it possible to exercise with good coordination, ensuring that the humeroscapular rhythm was close to normal. The HD group performed 11 exercises, each comprising three sets of 30 repetitions, three times a week for three months for a total of 36 treatments. Prior to the semiglobal and local exercises the HD patients warmed up for 15-20 minutes on an ergometer cycle. Half way through the exercise program (four exercises each of three sets of 30 repetitions) the patients cycled for 10 minutes. After the last four exercises, the patients spent another 10 minutes on the stationary ergometer cycle. The intensity during cycle exercises was moderate to high, i.e. a heart-rate frequency of $70-80 \%$ of the maximal heart rate. Patients in the LD group performed a total of six exercises, starting each treatment with five to ten minutes on an ergometer cycle, and then performed five semiglobal and local exercises using medical exercise therapy equipment, performing two sets of 10 repetitions of each exercise. The intensity during cycle exercises was moderate to high, as in the HD group, i.e. a heart-rate frequency of $70-80 \%$ of the maximal heart rate. Mean time for every treatment was approximately 30 minutes in the LD group and approximately 60 minutes in the HD group. All patients in both groups were treated over the three-month period with three treatments a week. For all patients a physiotherapist was present in the exercise room motivating, supporting, and observing the exercises according to the patients' clinical presentation and change in function and symptoms over the three-month treatment period. Thus, all patients received the same amount of attention while they were in the exercise room. The variables that were continuously observed were range of motion and weight resistance, while the number of exercises, repetitions, sets, and time spent on performing aerobic work using a stationary bike were kept constant in both groups.

\section{Data Analysis}

The statistical analysis was performed using the commercial software package SPSS for Windows (release 17). The significance level was set at $5 \%$, with a power of $90 \%$ and an SD of 3.2 points for pain, as presented by Conroy and Hayes [17]. To detect 2-point differences on the visual pain scale, a minimum of 27 patients was required for each group. Estimating a possible loss of $10 \%$ of the patients, we determined that each group would contain 30 patients. The age variable was considered and had normal distribution. The student's t-test for independent samples was used. The groups were compared for improvements in the mean. The Spearman's correlation test was used to assess correlation between variables.

\section{RESULTS}

Ninety-one patients were referred to the physical therapists from the general practioners, and thirty patients were excluded. Sixty-one patients (31 in the HD and 30 in the LD groups) were included in the study. Ninety-two percent of the patients completed the three-month intervention in the study. Five patients were lost to posttest; two in the HD group and three in the LD group. In the HD group, one patient moved away from the city and was therefore unable to keep in touch, and the second HD subject withdrew after experiencing a new injury at work that interfered with the continuation of the exercise treatment. In the LD group one subject was referred by his physician for additional outpatient therapy and therefore withdrew from the study. Two other patients in the LD interventions were not able to return for the posttest for personal reasons. Another four patients were lost to follow-up, two in the HD group and two in the LD group (Fig. 1).

In the HD group both pain and function were significantly improved from pre- to posttest $(p<0.05)$ compared to the LD group. The mean values of changes in range of motion and isometric force score, at pre- and posttraining periods in the HD and LD groups are plotted in Table 3 with confidence intervals. From pretest to follow-up there were no changes compared to pretest to posttest (Table 4).

In the HD group there were significant correlations $(\mathrm{p}<$ 0.01 ) from pretest to both posttest and follow-up between the following variables: pain and function, pain and external rotation, pain and abduction (strength), pain and abduction (ROM), function and external rotation, and flexion (strength). 


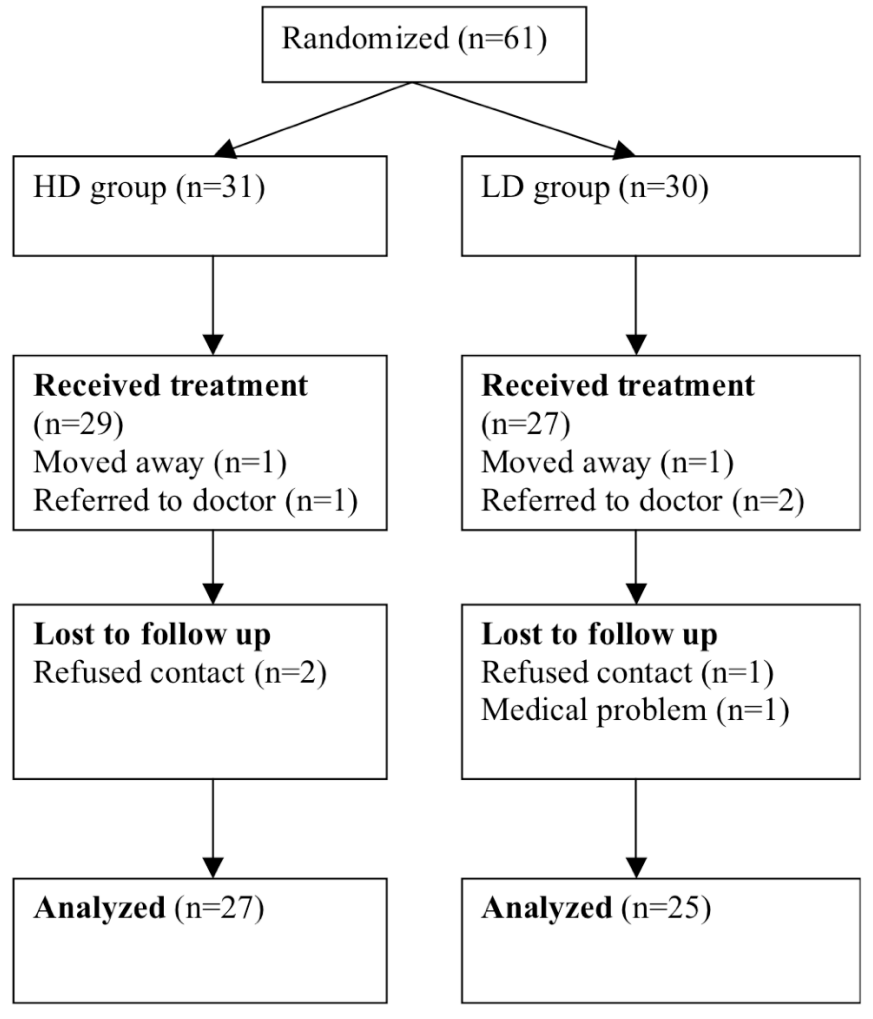

Fig. (1). Subject flow diagram.

\section{DISCUSSION}

Our objective was to investigate the effects of medical exercise therapy on patients with shoulder impingement syndrome. Our study is the first to use the medical exercise therapy method [16] on patients with shoulder impingement syndrome. A number of reviews have studied clinical effects of various exercise regimens in patients with rotator cuff tendinopathy or impingement syndrome [4]; however, the interventions are often not described or are low dosage.

Some studies of primary (without glenohumeral joint instability) shoulder impingement syndrome have used exercises $[18,19]$, but most failed to describe the exercises in detail in terms of intensity, duration, frequency, and load. In the present study we determined such factors in a shoulder girdle strengthening program for the shoulder musculature based on medical exercise therapy. But we also included a rather high total dose of global circulation exercises; which is not reported elsewhere in the literature. Thus, it is difficult to compare the results with those from other studies. Our results demonstrate that there was a statistically significant improvement in pain (assessed using a visual analog scale) favoring the HD group over the LD group and also demonstrate that medical exercise therapy aiming to activate the musculature of the affected shoulder was effective in increasing range of motion and strength. To our knowledge, the present work is the first to report a strong correlation between primary outcome (pain) and impairment (strength and ROM).

Ginn et al. [13] reported improvements in function and ROM with pain-free abduction and flexion among patients with nonspecific shoulder pain, regardless of etiology. Individualized treatment was carried out with strengthening, stretching, and scapulohumeral rhythm exercises. They reported no improvement in pain between the exercise group and control group. However, there was an improvement in the abduction ROM in the group that underwent exercise training. The results from our study revealed an improvement in pain, thereby differing from Ginn et al. results [13], while corroborating the results of other studies $[20,21]$. Our study also found improvement in the abduction ROM in the group treated with exercise in comparison to the control group, which agrees with other studies $[13,21]$. This demonstrates that such movement is considerably compromised in shoulder injuries and can be improved with exercise.

The development of chronic shoulder pain - here the patients had pain for as long as 3.6 and 3.1 years (HD and LD, respectively) - may lead to fear avoidance [22]. It is well documented that pain-related fear also affects physical performance and the overall physical activity level [23].

Table 3. Changes from Baseline to End of Treatment Among 56 Patients with Subacromial Impingement Syndrome, with Intention-to-Treat Analysis. Values are Mean (95\% Confidence Interval) According to One Analysis of Variance

\begin{tabular}{|c|c|c|c|}
\hline Variables & High-Dosage Exercise Group $(n=29)$ & Low-Dosage Exercise Group $(n=27)$ & p-Value \\
\hline Function (SRQ: $17=$ worse, $90=$ best) & $25.7(19.1$ to 32.1$)$ & $7,7(4.5$ to 10.9$)$ & 0.01 \\
\hline Satisfaction $(2=$ worse, $10=$ best $)$ & $3.9(2.1$ to 5.7$)$ & $2,1(0.9$ to 3.2$)$ & 0.03 \\
\hline - Flexion & $31(7$ to 40$)$ & $7(-2$ to 20$)$ & 0.01 \\
\hline - Abduction & $42(26$ to 54$)$ & $12(-2$ to 19$)$ & 0.01 \\
\hline \multicolumn{4}{|l|}{ Isometric Strength $(\mathbf{N})$} \\
\hline - Abduction & $34(24$ to 44$)$ & 17 (9 to 24$)$ & 0.03 \\
\hline - Flexion & $33(20$ to 41$)$ & $4(-1$ to 10$)$ & 0.07 \\
\hline
\end{tabular}


Table 4. Change from Baseline to 6 Months After End of Treatment Among 56 Patients with Subacromial Impingement Syndrome, with Intention-to-Treat Analysis. Values are Mean (95\% Confidence Interval) According to One Analysis of Variance

\begin{tabular}{|c|c|c|c|}
\hline Variables & \multicolumn{2}{|c|}{ Change in Score } & p-Value \\
\hline Function (SRQ: $17=$ worse, $90=$ best) & $29.2(21.7$ to 36.0$)$ & $7.3(0.2$ to 19.7$)$ & 0.01 \\
\hline Satisfaction ( $2=$ worse, $10=$ best $)$ & $4.2(3.6$ to 4.8$)$ & $2.0(1.6$ to 2.9$)$ & 0.03 \\
\hline - abduction & $49(33$ to 65$)$ & $14(-2$ to 24$)$ & 0.01 \\
\hline \multicolumn{4}{|l|}{ Isometric Strength (N) } \\
\hline - abduction & $45(28$ to 62$)$ & $14(-5$ to 29$)$ & 0.02 \\
\hline - flexion & $49(25$ to 65$)$ & $28(2$ to 41$)$ & 0.07 \\
\hline
\end{tabular}

VAS; visual analogue scale, SRQ; shoulder rating questionnaire.

Over time, this may have the negative consequence of decreasing the daily activity level, possibly resulting in functional incapacity. In the present study, it was emphasized that the exercises should be performed pain free or with as little discomfort as possible. Fearful patients might benefit from graded exposure to movements and activities that they previously avoided. However, we had no control of this factor among the included patients, since no instrument was used. We had a difference in the time spent at the treatment centre (Table 2) and hereby the level of attention and time from the physical therapists was higher in the HD group. This is a factor that could have influenced the results. We suggest that one possible mechanism behind the significant difference in the present study is that the HD group performed a considerable amount of global endurance exercises, reducing fear avoidance through pain reduction. This is a finding that should be examined in further research.

Lombardi et al. [24] investigated the clinical effects of a progressive resistance training program in impingement patients for the musculature of the shoulder. The program was held twice a week for two months, while the control group remained on a waiting list. Interestingly, they found no improvement in muscle strength among the patients treated, despite the clinical improvement. They explain this as possibly being due to the short period of intervention. The improvement in function may be related to an improvement in the pain [24]. One explanation is that when there are limitations in the performance of activities, patients with shoulder pain find ways to adapt, which may lead to a better result in terms of functional activity. There were quite many exclusion criteria in the present study, and the intervention - particularly the HD - requires that the patients are able to perform quite a lot of training. From a clinical practice point of view, it may reduce the number of subject that could receive such treatment. However, it might be possible to adjust the amount of training to each patient's ability, increasing the total amount of training over many weeks. Treatment like the HD program in the present study also requires good communication skills from the therapists to motivate the patients.
We may need a paradigm shift away from the belief that strengthening the lower trapezius is the solution, and away from the belief that specific exercises for a specific muscle in the shoulder can be the answer to the problem. Concurring with Battaglia-Mayer et al. [25], we suggest that further studies should include a better understanding of the handshoulder-arm function and of the fact that we are dealing with an extremity with complex neuromuscular functions that serve the hand. It is the function of the hand which is essential for normal shoulder function, including the lower trapezius and the rotator cuff. The biomedical view of the function of the supraspinatus muscle is that it abducts, externally rotates, and together with the other rotator cuff depresses the glenohumeral joint. According to the World Health Organization's (WHO) International Classification of Functioning, Disability and Health (ICF) [26], another approach is presented; the task of the supraspinatus is to work with the other rotator cuff muscles, the shoulder girdle muscles, and the trunk and arm muscles to position the hand to perform a functional activity. This view has been applied when choosing the eight different semiglobal and local exercises performing three sets of 30 repetitions of each exercise, along with three global aerobic circulation exercises.

One limitation in the present study is that the measurements were not undertaken by another person than the treating physiotherapists. However, this was a multicenter study with four physiotherapists. Nevertheless, as the outcome measurements were not obtained by a blinded assessor, this is a major limitation in this study, as blinded assessment is considered essential for preventing bias and assuring internal validity in a clinical trial. Further studies should be performed with a blinded tester. Another limitation is that there might have been additional rotator cuff or labral pathology that was not discovered by the clinical examination. An additional radiographic examination might have been preferable. Most literature on exercise treatment does not document the dose or type of exercises used very well, and this may be part of the explanation for 
the contradictory effects. Further, in all muscle strength tests, there was some pain in some of the patients. This is important for the interpretation, since if pain was present, then it was not the level of strength that was measured, rather pain tolerance. When evaluating efficacy of treatments, it is important to consider the patients' expectations since it might influence the results. However, the patients in the present study the patients did not have any treatment preference.

There is a great need for further research in the field of dose-response in therapeutic rehabilitation. Emphasis should be on clinical trials comparing difference rehabilitation protocols. Further trials should be adequately powered and undertake address blinding of outcome assessors. In spite of the mentioned methodological limitations, we claim that the present study has a certain amount of generalizability. However, the most important finding may be that the results shed some light on the issue of dose-response effects in treatment of shoulder impingement.

\section{CONCLUSION}

The purpose of this randomized controlled clinical trial was to compare two different exercise regimes in nonoperated patients with subacromial pain syndrome. In conclusion we found a dose-response effect of medical exercise therapy for twelve weeks in non-operated patients with longstanding subacromial pain syndrome as compared to low-dose training. The health professions should be aware that there is a dose-response effect using medical exercise therapy treating patients with longstanding subacromial pain. These results clearly show that pain reduction gives an effect on such impairment variables as strength and ROM. Further studies on the use of this high-dose regimen could be undertaken in comparison with surgery.

\section{ACKNOWLEDGEMENTS}

The authors are greatly thankful to the physical therapists Lasse Haugerud and Berit Østerås for their cooperation in performing the intervention. The authors received no funding in the writing or preparation of this manuscript and have no conflicts of interest.

\section{REFERENCES}

[1] Van der Windt DA, Koes BW, de Jong BA, Bouter LM. Shoulder disorders in general practice: Incidence, patient characteristics, and management. Ann Rheum Dis 1995; 54: 959-64.

[2] Jette AM, Delitto A. Physical therapy treatment choices for musculoskeletal impairments. Phys Ther 1997; 77: 145-54.

[3] Luime JJ, Koes BW, Hendriksen IJ, et al. Prevalence and incidence of shoulder pain in the general population; A systematic review. Scand J Rheumatol 2004; 33(2): 73-81.

[4] Kuhn JE. Exercise in the treatment of rotator cuff impingement: A systematic review and synthesized evidence-based rehabilitation protocol. J Shoulder Elbow Surg 2009; 18: 138-60.

[5] Desmeules F, Cote CH, Fremont P. Therapeutic exercise and orthopedic manual therapy for impingement syndrome: a systematic review. Clin J Sport Med 2003; 13: 176-82.
[6] Michener LA, Walsworth MK, Burnet EN. Effectiveness of rehabilitation for patients with subacromial impingement syndrome: a systematic review. J Hand Ther 2004; 17: 152-64.

[7] Torstensen TA. The physical therapy approach. In: Frymoyer JW, Ducker TB, Hadler MN, Kostuik JP, Weinstein JN, Whitecloud TS, Eds. The adult spine: principles and practice. $2^{\text {nd }}$ ed. Philadelphia: Lippincott-Raven 1997: 1797-805.

[8] Beaudreuil J, Nizard R, Thomas T, et al. Contribution of clinical tests to the diagnosis of rotator cuff disease: A systematic literature review. Joint Bone Spine 2009; 76(1): 15-9.

[9] Huskisson EC. Measurement of pain. Lancet 1974; 2(7889): 1127-31.

[10] L'Insalata JC, Warren RF, Cohen SB. A self-administered questionnaire for shoulder assessment of symptoms and function of the shoulder. J Bone Joint Surg Am 1997; 79: 738-48.

[11] Williams JW, Holleman DRJ, Simel DL. Measuring shoulder function with the shoulder pain and disability index. J Rheumatol 1995; 22: 72732 .

[12] MicroFET2 Dynamometer, Westloch, USA.

[13] Ginn KA, Herbert RD, Khouw W, Lee R. A randomized, controlled clinical trial of a treatment for shoulder pain. Phys Ther 1997; 77: 80211.

[14] Kwoh CK, Petrick MA, Munin MC. Inter-rater reliability for function and strength measurements in acute care hospital after elective hip and knee arthroplasty. Arthritis Care Res 1997; 10: 128-34.

[15] Torstensen TA, Meen HD, Stiris M. The effects of medical exercise therapy on a patient with chronic supraspinatus tendinitis. Diagnostic ultrasound - tissue regeneration: a case study. J Orthop Sports Phys Ther 1994; 20(6): 319-27.

[16] Torstensen TA. Medical exercise therapy for shoulder, elbow and wrist pain - dysfunction of the upper extremity - Exercise manual. Oslo, Norway: Holten Institute 1999.

[17] Conroy DE, Hayes KW. The effect of joint mobilization as a component of comprehensive treatment for primary shoulder impingement syndrome. J Orthop Sports Phys Ther 1998; 28: 3-14.

[18] Haahr JP, Ostergaard S, Dalsgaard J, et al. Exercises versus arthroscopic decompression in patients with subacromial impingement: A randomised, controlled study in 90 cases with a one year follow up. Ann Rheum Dis 2005; 64: 760-4.

[19] Dickens VA, Williams JL, Bhamra MS. Role of physiotherapy in the treatment of subacromial impingement syndrome: a prospective study. Physiotherapy 2005; 91: 159-64.

[20] Winters JC, Sobel JS, Groenier KH, Arendzen HJ, Meyboom-De Jong B. Comparison of physiotherapy, manipulation, and corticosteroid injection for treating shoulder complaints in general practice: Randomised, single blind study. BMJ 1997; 314: 1320-5.

[21] Wang SS, Trudelle-Jackson EJ. Comparison of customized versus standard exercises in rehabilitation of shoulder disorders. Clin Rehabil 2006; 20: 675-85.

[22] Vlayen JWS, Linton SJ. Fear avoidance and its consequences in chronic musculoskeletal pain: A state of the art. Review article. Pain 2000; 85: 317-32.

[23] Crombez G, Vervaet L, Lysens R, Baeyens F, Eelen P. Avoidance and confrontation of painful, back straining movements in chronic back pain patients. Behav Modification 1998; 22: 62-77.

[24] Lombardi I Jr, Magri AG, Fleury AM, Da Silva AC, Natour J. Progressive resistance training in patients with shoulder impingement syndrome: a randomized controlled trial. Arthritis Rheum 2008; 59(5): 615-22.

[25] Battaglia-Mayer A, Caminiti R, Lacquaniti F, Zago M. Multiple levels of representation of reaching in the parieto-frontal network. Cereb Cortex 2003; 13(10): 1009-22.

[26] Sampaio RF, Luz MT. Human functioning and disability: exploring the scope of the World Health Organization's international classification. Cad Saude Publica 2009; 25(3): 475-83. 